\title{
Negative information: Both presence and absence of spatial pattern elements guide rats' spatial choices
}

\author{
ELIZABETH DiGELLO, MICHAEL F. BROWN, and JOHN AFFUSO \\ Villanova University, Villanova, Pennsylvania
}

\begin{abstract}
Rats learn the spatial pattern in which hidden caches of food are located. Once the pattern is learned, finding one or more baited locations provides (positive) information about the remaining baited locations. In the present experiment, we examined whether negative information (the absence of food in a location) would also be used in locating the remaining baited locations. Rats were tested in an apparatus containing a matrix of vertical poles, on top of which food could be hidden. At the beginning of the trial, the location of the baited poles was unpredictable, but the poles were always arranged in a linear pattern. The rats learned the pattern and used both positive and negative information in locating baited poles.
\end{abstract}

Information is often conveyed by the presence of a stimulus. However, there are also situations in which information can be conveyed by the absence of a stimulus. An example of such "negative" information from the study of classical conditioning is conditionedinhibition(e.g., Miller $\&$ Spear, 1985), in which the absence of an unconditioned stimulus affects future responding to conditioned stimuli. In the area of human perception and detection, differences in recognition of changes produced by the introduction of a new stimulus versus the deletion of a familiar one have been reported (e.g., Agostinelli, Sherman, Fazio, \& Hearst, 1986; Nallan et al., 1994). The use of information about absence or nonoccurrence has also been well studied in the areas of human judgment (Fiedler, 2000; Nisbett \& Ross, 1980) and deductive logic (Klayman \& Ha, 1987). Generally, a variety of approaches that involve negative information suggest that, although humans and animals are sensitive to negative information, the psychological effects of positive information (conveyed by the presence of a stimulus) are larger than the psychological effects of negative information (conveyed by the absence of a stimulus), even when the logical implications of the two types of information are equal.

This project was supported by National Science Foundation Grant BNS-9982244. The experiment was the basis of a thesis submitted to Villanova University by E.D. for the degree of Master of Science. E.D. is currently at the University of Illinois. We thank the reviewers for their insightful comments, which substantially influenced the paper. Correspondence should be addressed to M. Brown, Department of Psychology, Villanova University, Villanova, PA 19085 (e-mail: michael.brown@ villanova.edu).
Direct comparisons of the effectiveness of information conveyed by the presence of a stimulus and information conveyed by the absence of a stimulus have been made in the study of discrimination learning. Jenkins and Sainsbury (1969) first investigated the superiority of discrimination learning when the discriminating feature is present on reinforced trials, as opposed to absent on reinforced trials. They found that pigeons learned a discrimination faster when reinforcement occurred after presentation of a stimulus with a unique feature than when the same stimuli were used, but the stimulus without the unique feature was reinforced. This phenomenon was labeled the feature positive effect (FPE). Two aspects of the FPE are of interest for the present purposes. First, discrimination performance is superior when the positive information (unique feature) corresponds to the positive (reinforced) stimulus. Second, discrimination performance is supported by negative information, albeit not to the same degree (see Hearst, 1984, for a review of the FPE and related phenomena).

The present experiment was designed to examine the role of negative information in a spatial pattern learning task developed by M. F. Brown and Terrinoni (1996). In this task, rats search for food hidden on the tops of baited poles. The poles in the present experiment form a $4 \times 4$ matrix. On any particular trial, four poles are baited. There is no information about the identity of the baited poles prior to each trial. However, the baited poles are always arranged in a linear spatial pattern (one row of the matrix for some rats and one column of the matrix for the remaining rats).

This paradigm has been used in prior experiments in our laboratory to examine control of rats' choices by several spatial patterns, including a square pattern (M. F. Brown \& Terrinoni, 1996; Lebowitz \& M. F. Brown, 1999) and a 
checkerboard pattern (M. F. Brown, Zeiler, \& John, 2001). M. F. Brown and Terrinoni also found evidence for control by a linear pattern equivalent to the one used in the present experiment. C. R. Brown (1998) found some evidence that rats could be controlled by a more complex pattern consisting of one baited row and one baited column of a matrix. M. F. Brown, DiGello, Milewski, Wilson, and Kozak (2000) showed that rats can learn a conditional spatial pattern discrimination, in which the pattern of baited poles is either a square or a line, depending on the identity of the food with which the poles are baited.

In all of these experiments, we have focused on the information provided by discovery of a baited pole. At the beginning of each trial, there is no information about which poles are baited. However, because the poles are baited in a consistent pattern, once one or more baited poles are discovered, they potentially provide information about the location of remaining poles. For example, if the poles are always baited in a row, discovery of a baited pole specifies that the remaining baited poles are in the same row. Our results to date make it clear that rats learn to use this positive information conveyed by baited poles to locate remaining baited poles.

\section{Row Group}
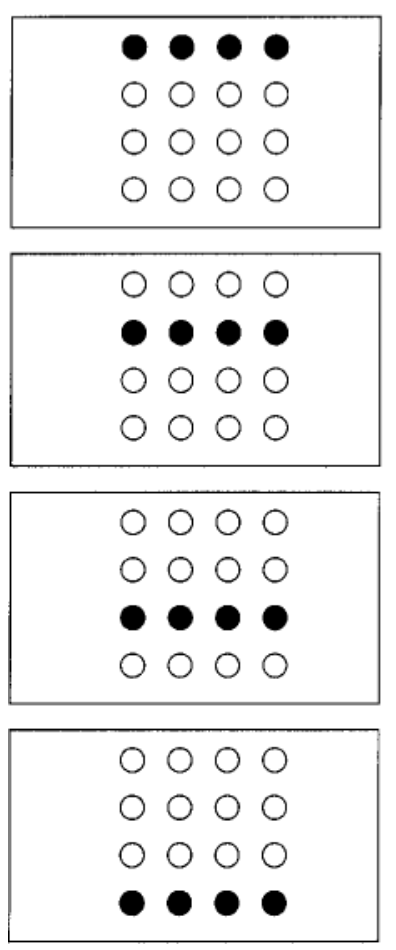

Figure 1. Schematic illustration of the four configurations of baited poles used for rats in each of the two groups. Baited poles are represented by filled circles and unbaited poles are represented by unfilled circles. For each group, the configuration used on each trial was unpredictable at the beginning of each trial. Illustration is not to scale.
For at least some patterns, however, information about the location of baited poles might also be conveyed by determination that a pole is not baited. For example, if the poles are always baited in a row, choice of an unbaited pole specifies that the baited poles are in a different row. The primary goal of the present experiment was to determine whether spatial pattern learning results in choices' being controlled by this negative information.

In the experiment, a line pattern in a $4 \times 4$ pole box was used. For half of the rats, the four baited poles were always in one of the four rows of the matrix. For the remaining rats, the four baited poles were always in one of the four columns of the matrix (Figure 1). Consider a rat exposed to the row pattern (Figure 2). If the rat happens to choose a baited pole, the locations of the remaining baited poles can be determined. The three remaining baited poles must be in the same row as the first baited pole just chosen. Our previous work suggests that rats will come to be controlled by this positive information. If the rat chooses an unbaited pole, information about the location of the remaining baited poles is also available-that is, they are in one of the three rows not containing the chosen pole. Will choices come to be controlled by this negative information?

\section{METHOD}

\section{Column Group}
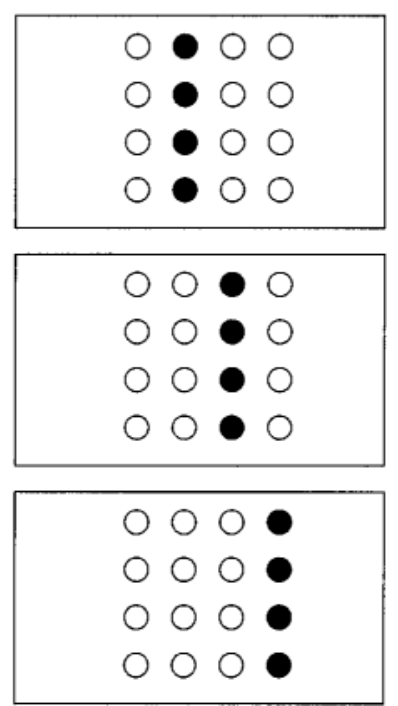

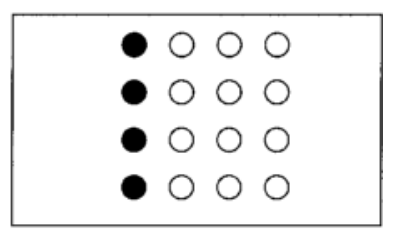

\section{Subjects}

Nineteen male Sprague-Dawley rats served as subjects. Two weeks before the start of the experiment, the rats were placed on a food-restricted diet. Each rat was given approximately $12 \mathrm{~g}$ of food (Purina Rat Chow) every day. This amount was adjusted in order to keep the rats' weight at $85 \%$ of normal free-feeding weight. Normal growth changes were calculated by using growth charts provided by the vendor (Harlan Sprague Dawley, Inc., Indianapolis). The rats had ad-lib access to water. They were housed in a 12:12-h reverse light:dark cycle and were tested during the dark phase. The rats were approximately 4 months old at the start of the experiment.

\section{Apparatus}

A pole box with a $4 \times 4$ matrix of poles was used in the experiment. The poles were constructed of two main components. First, unpainted, wooden dowels $(15.5 \mathrm{~cm}$ tall and $1.2 \mathrm{~cm}$ in diameter) were mounted to the floor of the pole box. A $0.5-\mathrm{cm}$ deep well was drilled into the top of each pole to allow for one sucrose pellet to be placed inside. (All 16 poles had this sham bait as a control for odor cues: The sham bait was replaced once a week.) Second, a section of PVC tubing $(15.5 \mathrm{~cm}$ tall and $2.2 \mathrm{~cm}$ in diameter) was placed over the wooden dowels. A PVC sleeve $(2.9 \mathrm{~cm}$ in diameter; normally used to connect two sections of tubing) held in place a piece of nylon mesh $1.0 \mathrm{~cm}$ from the top of the sleeve, which extended the height of the pole to a total of $16.5 \mathrm{~cm}$. The sham bait was under this mesh and was therefore inaccessible. The poles were baited by placing a sucrose pellet on top of the mesh, so that they were hidden inside the PVC sleeve $(1.0 \mathrm{~cm}$ from the top edge of the sleeve). The poles were $13 \mathrm{~cm}$ apart, and the matrix of poles was centered in the box, which was $120 \times 58 \times 33 \mathrm{~cm}$ (tall), built out of plywood and painted flat black. Bedding material covered the floor of the pole box.

A separate training apparatus was used during the training phase of the experiment. This apparatus consisted of four poles of four different heights: $2.0,4.5,12.5$, and $15.5 \mathrm{~cm}$. The poles were separated by $7 \mathrm{~cm}$ and arranged on a piece of cardboard in a straight line in increasing order of height. The training apparatus could easily be placed in and removed from the pole box. 

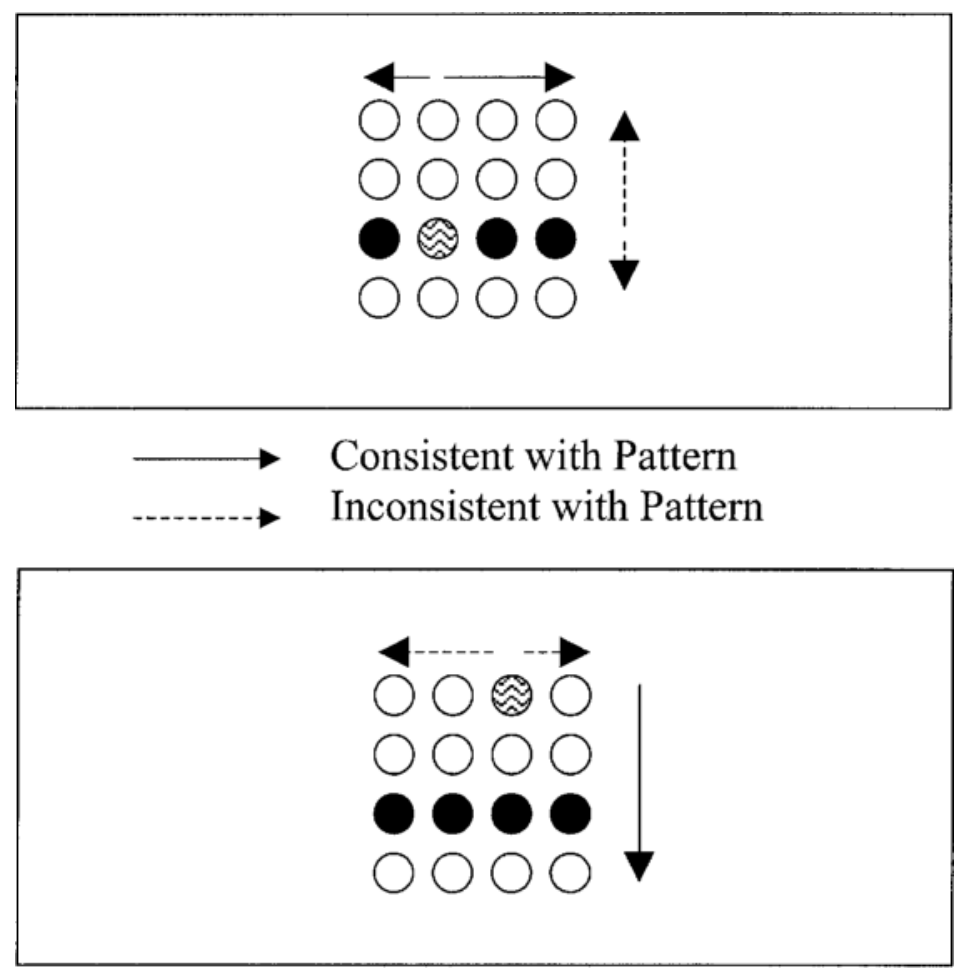

Figure 2. Illustration of the logic of measuring choices consistent and inconsistent with the spatial pattern used in the experiment. The example is a rat in the row group, during a trial in which the third row of poles is baited (filled circles represent baited poles). The top panel shows the case in which a rat has just chosen a baited pole (stippled circle represents the just-chosen pole). Choices of poles in the same row as the just-chosen pole would be consistent with the pattern; choices of poles in the same column as the just-chosen poles would be inconsistent with the pattern. The bottom panel shows the case in which a rat has just chosen an unbaited pole. Choices of poles in the same column as the just-chosen pole would be consistent with the pattern; choices of poles in the same row as the just-chosen pole would be inconsistent with the pattern. The converse contingencies apply to rats in the column group (not shown).

\section{Procedure}

For 3 days before the start of the experiment, the rats received 1020 sucrose pellets in their home cage. Training began following the 3rd day. Access to the $4 \times 4$ matrix of poles was blocked by a wooden barrier, and the training apparatus was placed in one of the open ends of the box. The training poles were baited with sucrose pellets, and additional pellets were placed around the training apparatus for the first two trials. The rat was placed in the end of the box containing the training apparatus and was allowed to find the pellets. A training trial ended when the rat had found all the pellets or $8 \mathrm{~min}$ had elapsed. Training ended for an individual rat when he had eaten all the pellets on two consecutive trials. One training trial was conducted per day.

After successful training, the test trials began. Ten rats were assigned to the column pattern. For these rats, one of the four columns of poles was baited on each trial. The remaining rats were assigned to the row pattern. For these rats, one of the four rows of poles was baited on each trial. The specific column or row baited on each trial was counterbalanced in blocks of 12 trials (three trials with each column, or row, per block).

A rat was placed in one end of the pole box at the start of each trial. The trial ended when the rat had found all the baited poles or when $5 \mathrm{~min}$ had elapsed. The experimenter recorded the rat's choices as the animal moved through the box. A choice was recorded when the rat's nose came within $1 \mathrm{~cm}$ of the top of the pole while the rat was rearing. This definition of a choice was developed in earlier experiments in which the same apparatus was used and has a very high interrater reliability even when raters are not aware of the identity of the baited poles (99.1\% interrater agreement in M. F. Brown et al., 2000, Experiment 2). All of the trials were videotaped so that any ambiguous choices could be resolved (this was needed very rarely). A total of 100 experimental trials were run with each rat. One or 2 trials were completed per day. When 2 trials per day were conducted, the trials were separated by at least $1 \mathrm{~h}$.

\section{RESULTS AND DISCUSSION}

The data were analyzed in four blocks of 25 trials each. All statistical decisions were made using a criterion for rejection of the null hypothesis of $p<.05$.

The first analysis examined the number of choices made leading up to (and including) the discovery of the first, second, third, and fourth baited pole located by the rat. Figure 3 shows the mean number of choices made in locating each of the four baited poles for rats with the row 


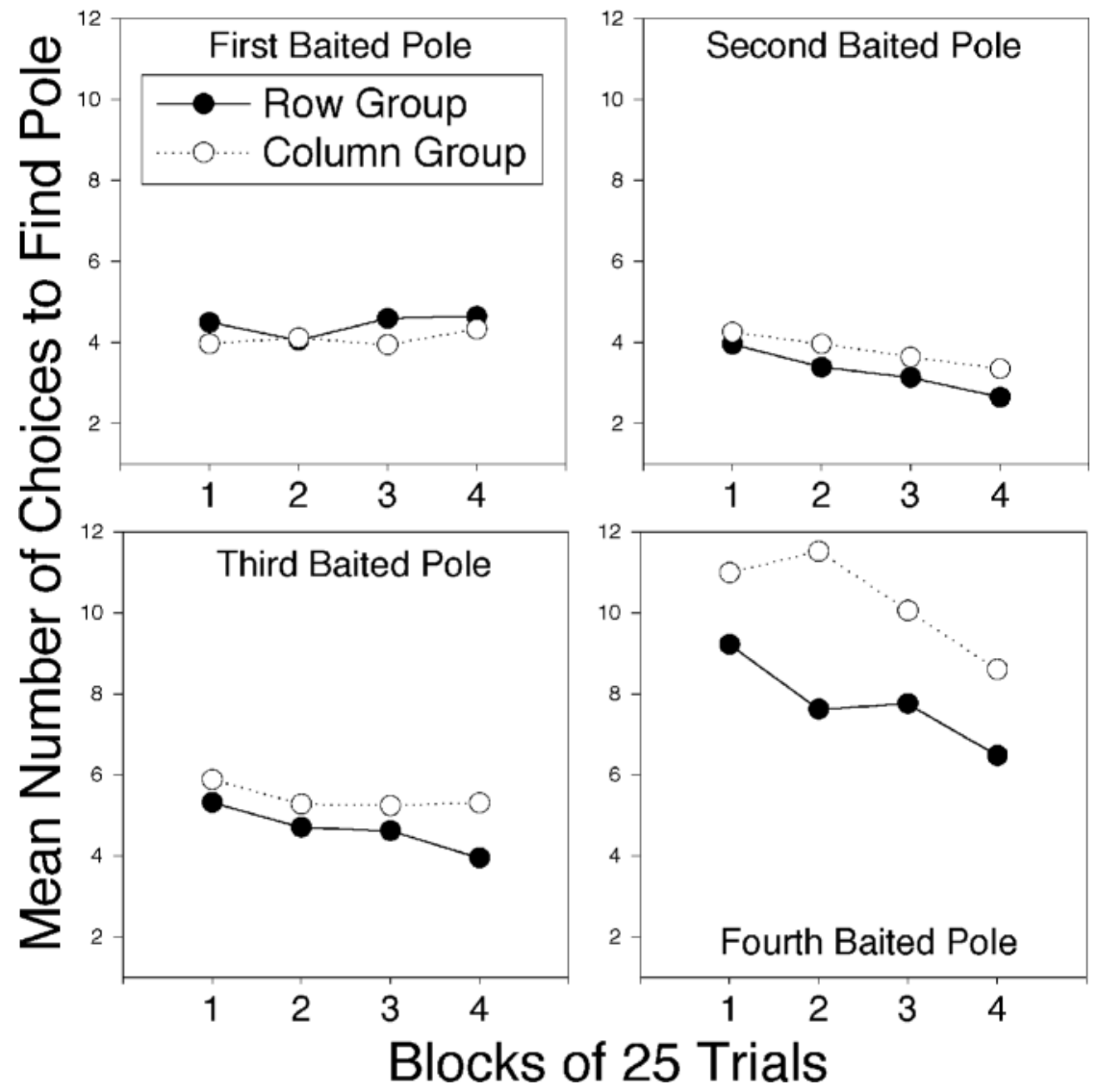

Figure 3. Mean number of choices taken to discover each baited pole in the sequence of four baited poles discovered during each trial for the two groups over four trial blocks. The value is the number of choices made since the last baited pole was discovered (or, in the case of the first baited pole, the number of choices made since the beginning of the trial).

pattern and column pattern over the four trial blocks. (Cases in which the rat did not discover all four baited poles before the end of trial resulted in slightly smaller $n$ s for later discoveries. The rats discovered all four baited poles by the end of the trial during means of $95 \%, 100 \%$, $96 \%$, and $96 \%$ of the trials in Blocks $1-4$, respectively.) The effects of the variables represented in this figure were evaluated with a group (rows vs. columns) $\times$ block $\times$ serial position of discovery analysis of variance (ANOVA). Differences in the number of choices required for locating baited poles as a function of the serial position of the discovery of that pole $[F(3,51)=162.2]$ are very difficult to interpret, because the proportion of poles in the matrix that are baited changes as a direct result of those discoveries. A critical result is that performance improved over trial blocks $[F(3,51)=9.5]$, showing that there was some change or changes in the choice behavior of the rats over blocks that resulted in improved choice accuracy. Spatial pattern learning is a possible mechanism of this change, but it is not the only possibility.

If the improvement in performance over blocks is due to an increased ability to use information about the pattern in which baited poles were arranged, the improvement should be larger for discoveries of the second, third, and fourth poles than for discovery of the first baited pole. No positive information is available about the locations of the baited poles until after the first baited pole is discovered. Thus, any contribution of spatial pattern learning to the ability to locate the first baited pole would depend exclusively on the use of negative information. After one or more baited poles have been discovered, on the other hand, both negative and positive information about the location of remaining poles is available.

An examination of Figure 3 suggests that the improvement in performance was in fact restricted to discoveries of poles following the first. The effect of blocks interacted with the effect of serial position of the discovery $[F(9,153)=3.04]$. The interaction resulted from there being an effect of block on the number of choices required to discover the second $[F(3,51)=4.87]$ and fourth $[F(3,51)=$ 5.58] baited poles but no evidence for an effect of block on the number of choices required to discover the first $[F(3,51)=1.81]$ or third $[F(3,51)=2.28, .05<p<.10]$ baited poles. 
Overall, the row group required fewer choices to discover the baited poles than did the column group $[F(1,17)=$ 35.6]. It is not clear how best to interpret this effect. One possibility is that it has to do with a thigmotaxic tendency of the rats to choose poles along the walls of the pole box. The walls parallel to rows were much closer to the pole matrix than were the walls parallel to columns (see Figure 1). Thus, this effect may be best explained as the result of rats' tending to sequentially choose poles in the outside rows.

It would be of interest to compare the performance levels shown in Figure 3 with those expected by chance. A literal estimate of chance performance assumes that each choice is an independent random selection from among the 16 poles and predicts that a mean of $4,5.34,8$, and 16 choices would be required to locate 1 of $4,3,2$, and 1 target pole(s), respectively (and thus these chance values correspond to the conditions represented in the four panels of Figure 3 ). These values suggest that the rats did no better than chance in locating the first baited pole, but located subsequent baited poles better than expected on the basis of chance. These estimates of chance must be interpreted with caution, however. They do not take into account many factors (aside from the control by the spatial pattern that is of central interest here) that might affect choice accuracy. For example, rats tend to choose poles in proximity to the most recent choice (M. F. Brown \& Terrinoni, 1996; M. F. Brown et al., 2001). There is a tendency to choose poles close to the walls of the apparatus (Lebowitz \& M. F. Brown, 1999). There is a tendency to avoid revisits to poles chosen earlier in the trial (Lebowitz \& M. F. Brown, 1999). These effects and the complex interactions among them make it difficult to isolate any contribution of spatial pattern from other choice processes that contribute to performance in this task. Thus, after an examination of pole revisits, the focus will be on a measure that is explicitly designed to isolate control of choices by the spatial pattern.

Measures of the tendency to avoid revisiting poles in the present experiment are helpful in understanding the choice accuracy levels shown in Figure 3 and are there-
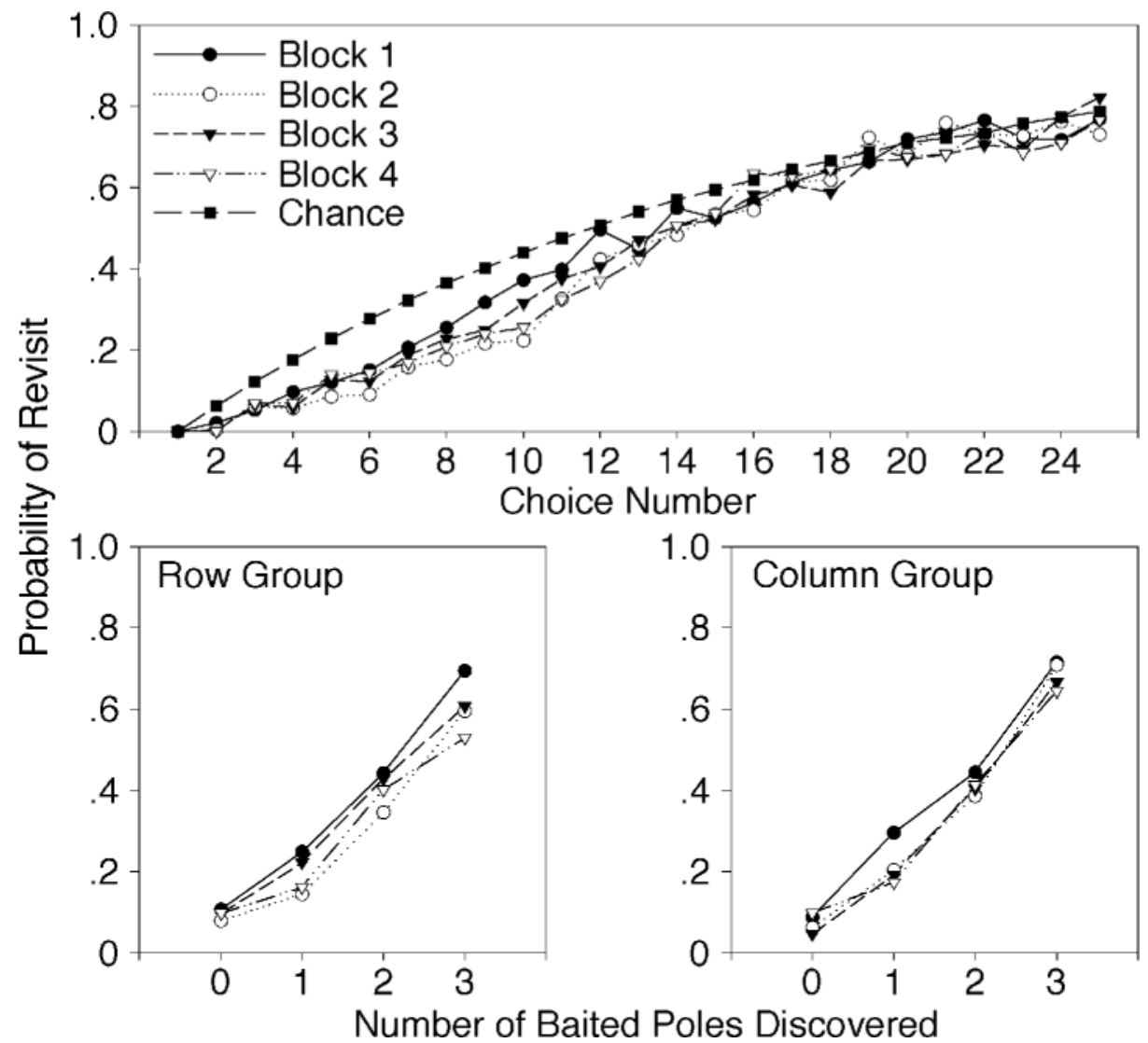

Figure 4. The mean (over rats) proportion of choices that were revisits of poles visited earlier in the trial. The top panel shows these values as a function of choice number (up to the 25 th choice of each trial; values for choices beyond Choice 4 are based on the subset of trials in which the rat made at least the corresponding number of choices). Also shown in the top panel is the proportion expected if rats chose randomly from among the 16 poles on each choice (the expected values are based on 10,000 iterations of a Monte Carlo simulation). The bottom panels show the same measure but as a function of the number of baited poles that had been discovered so far during the trial. 
fore shown in Figure 4. The top panel shows the probability of choosing a pole that had been previously visited during the trial, as a function of choice number (up to choice number 25) and trial block. Shown for comparison is the probability of a revisit expected if the rat chooses randomly from among the 16 poles on each choice. The bottom panels show these data in a form that may be more useful for the present purposes. Here the probability of revisiting poles is shown as a function of the number of baited poles that have been discovered, as well as trial block and experimental group. A group $\times$ trial block $\times$ number of poles previously discovered ANOVA indicated that the probability of a revisit differed as a function of trial block $[F(3,51)=11.0]$ (pairwise comparisons of the values for trial blocks indicated that Block $1>$ Block $2=$ Block $3=$ Block 4) and was, clearly, more prevalent as the trial progressed (number of poles previously discovered) $[F(3,51)=1,387.0]$. There was an interaction between the effects of these two variables $[F(9,153)=3.6]$. Thus, there is an ability to avoid revisits, which improves with experience. However, as the density of previously visited poles increases over the course of trials, revisits of poles become quite common. Certainly, the rats did not exhibit the very strong tendency to avoid revisits that has been found in some other spatial choice paradigms, such as the radialarm maze (Olton \& Samuelson, 1976). This fact is important in understanding the relatively low levels of choice accuracy shown in Figure 3 (and in the pole box task, generally) in comparison with performance in other commonly used spatial tasks.

The central analysis of performance in this experiment was designed to focus on the use of information based on learning of the spatial pattern and obtained from choosing a baited or unbaited pole. Choices were classified according to the spatial relation between the pole from which the rat moved and the pole to which the rat moved during each choice. Choices were eliminated from the analysis if either of the two poles involved had been visited previously during the trial. This eliminates the effects of tendencies the rats have to avoid revisits and avoids ambiguity about the status of a previously baited but already visited pole from which the rat initiates a choice. All choice transitions that did not involve a previously visited pole were classified into one of three categories: (1) moves from a pole to a pole in the same row, (2) moves from a pole to a pole in the same column, and (3) other moves (i.e., moves to a pole that was in a different row and a different column).

To the extent that rats learn the row or column pattern and that learning is expressed by pole choices, the distribution of choices in these categories should vary as a joint function of the orientation of the pattern (row vs. column) and the status of the pole from which the rat moves (baited vs. unbaited). In particular, rats in the row group should tend to move to poles in the same row after visiting a baited pole and to poles in the same column after visiting an unbaited pole, as is illustrated in Figure 2. The rats in the column group should exhibit the opposite tendencies. Note that the tendencies expected following the choice of a baited pole are dependent on positive information, derived from discovery of a baited pole. The tendencies expected following the choice of an unbaited pole are dependent on negative information, derived from choosing a pole that is not baited.

Figure 5 shows the tendencies of rats to make same-row and same-column moves. The tendencies appear to be as predicted. They were examined starting with a move type (row vs. column moves) $\times$ block $\times$ status of pole from which rat moved (baited vs. unbaited) $\times$ group ANOVA. Because a four-way interaction among the effects of these variables was found $[F(3,51)=7.05]$, the tendencies following choice of baited and unbaited poles were analyzed separately. This allowed behavior in response to positive and negative information to be isolated.

Row and column moves following discovery of a baited pole (top panels of Figure 5) were compared using a move type $\times$ block $\times$ group ANOVA. The critical result is a move type $\times$ group interaction $[F(1,51)=34.4]$ produced by the relatively greater tendency of the row group to make row moves and the column group to make column moves. This finding shows that the discovery of a baited pole served as a cue that allowed the rats to search in accordance with the pattern of baited rows or columns. There was also a significant triple interaction $[F(3,51)=$ 4.55], indicating that the tendencies to make choices that conformed to the pattern increased over blocks.

Row and column moves following the discovery of an unbaited pole (bottom panels of Figure 5) were compared using an analogous move type $\times$ block $\times$ group ANOVA. Again, the critical move type $\times$ group interaction was obtained $[F(1,51)=14.3]$, in this case produced by the relatively greater tendency of the row group to make column moves and the column group to make row moves following the choice of an unbaited pole. However, in this case, there was no significant triple interaction $[F(3,51)=1.7]$, and therefore no evidence that the tendency to make choices that conformed to the pattern increased over blocks. The move type $\times$ group interaction shows that finding no bait on a pole, just like finding a baited pole, serves as a cue that allows rats to make the next choice in accordance with the pattern.

The experimental task that we have been using to study spatial pattern learning in rats appears to involve two major sets of processes. First, the rat learns the spatial pattern in which the baited poles are arranged. Second, the rat locates baited poles on each trial using information about the learned pattern in combination with information about the status of visited poles (baited vs. unbaited). Previous work from our laboratory shows that rats use the positive information provided by discovery of a baited pole to locate additional baited poles in the context of square (M. F. Brown \& Terrinoni, 1996; Lebowitz \& M. F. Brown, 1999), linear (M. F. Brown et al., 2000), and checkerboard (M. F. Brown et al., 2001) patterns. The results of the present experiment confirm that finding for a linear pattern and extend it by showing that the choice of an unbaited pole as well as the choice of a baited pole provides infor- 


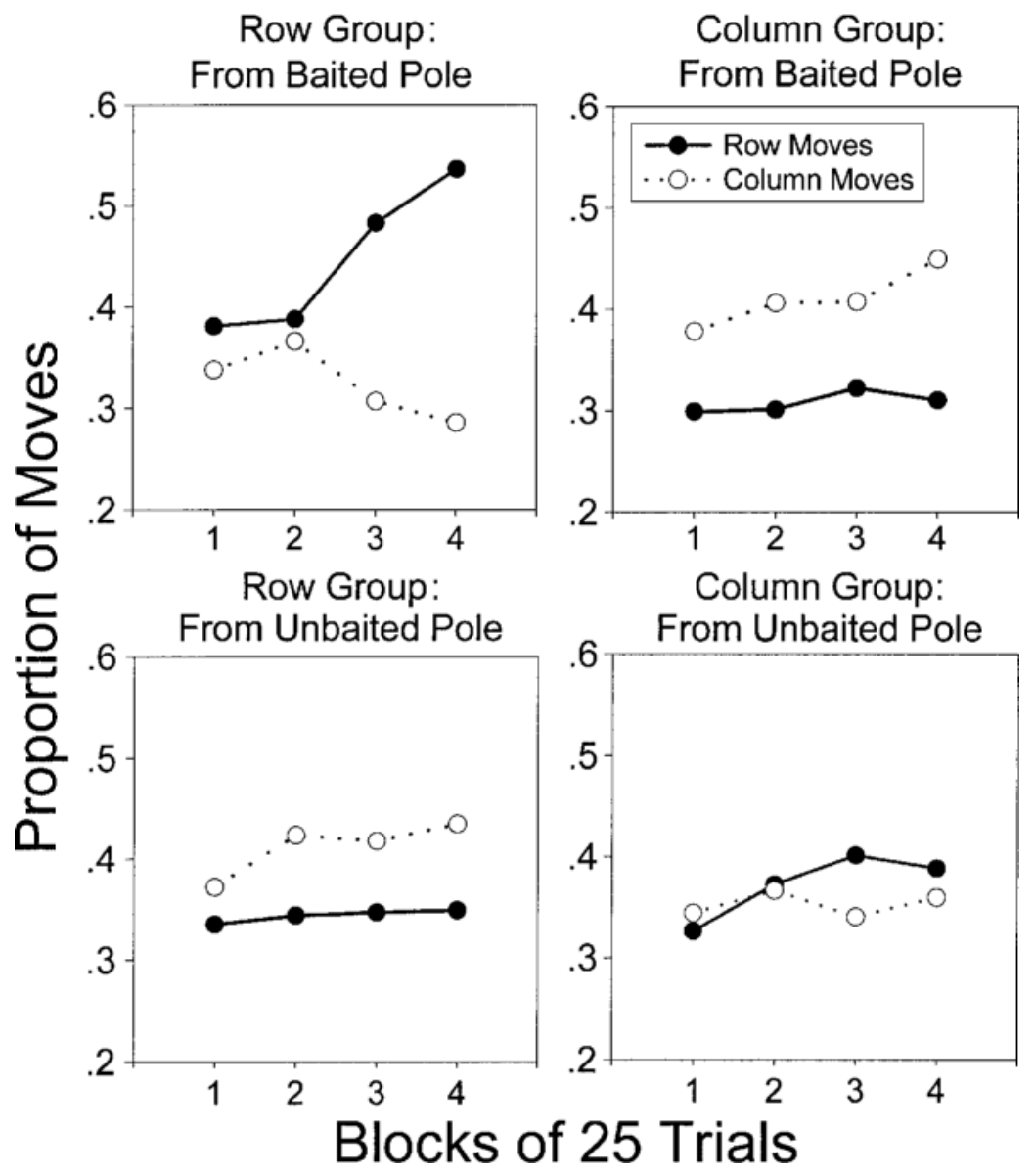

Figure 5. Mean proportion of choices that were to poles in the same row (row moves) or the same column (column moves) as the most recently chosen pole (although the tendencies to move to poles located in both a different row and different column are not shown, they can be determined from the figure because they include all remaining moves). Means are shown for the two groups as a function of whether the first choice of the two was to a baited or to an unbaited pole. Cases included in this analysis were restricted to those in which both choices were to poles that had not been previously visited.

mation about the location of the remaining baited poles. On the basis of our previous work, we have argued that a representation of the spatial relations among the baited poles forms during spatial pattern learning. Use of this negative information suggests that the representation of the spatial pattern acquired by the rat includes the spatial relations between the baited poles and the unbaited poles, as well as the spatial relations among the baited poles.

The control by negative information found in the present experiment appears to be weaker than the control by positive information. However, the data do not allow us to come to any strong conclusions with respect to the relative level of control by positive and negative information. Consider that, after a rat has visited an unbaited pole, a move to a pole in a different row and a different column (i.e., all the moves not represented by the proportions that are shown in Figure 5) is consistent with the negative information provided. Our formal comparisons are restricted to moves in the same column or same row as that of the just-visited pole, because this comparison provides control for distance and other spatial relations among the poles involved in the moves considered in the analysis. Given the necessity of control for these factors for making meaningful comparisons, we are not able to fully compare the effects of positive and negative information on spatial choice ability. However, our analysis provides clear evidence that both effects exist.

Although we cannot determine that negative information supports a lower level of control than does positive information on the basis of these data, the data do suggest that possibility, and such an asymmetry would be consistent with data from the other domains reviewed in the introduction. At least two general classes of explanations for a greater degree of control by positive information than by negative information are relevant in the context of the present experiment. One points to the asymmetry in the 
amount of information provided by positive and negative information in the present experimental paradigm (discovery of a baited pole specifies the location of the remaining poles, choice of an unbaited pole rules out 3 poles as possibilities for being baited, leaving 12 possibly baited poles of which 4 are baited). A second class of explanation stems from the possibility that there is a natural bias so that positive information has greater psychologicaleffects than does negative information, regardless of their relative formal validity (Hearst, 1984; Hearst \& Wolff, 1989).

As reviewed above, there are a number of domains in which the use of negative information has been studied in both human and nonhuman subjects. The present work extends the study of control by the absence of stimuli to the spatial domain. Additional work will be required in order to understand the mechanisms involved in the use of negative information about spatial location.

\section{REFERENCES}

Agostinelli, G., Sherman, S. J., Fazio, R. H., \& Hearst, E. (1986). Detecting and identifying change: Additions vs. deletions. Journal of Experimental Psychology: Human Perception \& Performance, 12, 445-454.

Brown, C. R. (1998). Spatial pattern learning by rats in the absence of landmarks. Unpublished master's thesis, Villanova University, Villanova, Pennsylvania.

Brown, M. F., DiGello, E., Milewski, M., Wilson, M., \& KozaK, M. (2000). Spatial pattern learning in rats: Conditional control by two patterns. Animal Learning \& Behavior, 28, 278-287.

Brown, M. F., \& Terrinoni, M. (1996). Control of choice by the spa- tial configuration of goals. Journal of Experimental Psychology: Animal Behavior Processes, 22, 438-446.

Brown, M. F., Zeiler, C., \& John, A. (2001). Spatial pattern learning in rats: Control by an iterative pattern. Journal of Experimental Psychology: Animal Behavior Processes, 27, 407-416.

FiEDLER, K. (2000). Beware of samples! A cognitive-ecological sampling approach to judgement biases. Psychological Review, 107, 659-676.

HeARst, E. (1984). Absence as information: Some implications for learning, performance, and representational processes. In H. L. Roitblat, T. G. Bever, \& H. S. Terrace (Eds.), Animal cognition (pp. 311332). Hillsdale, NJ: Erlbaum.

Hearst, E., \& WolfF, W. T. (1989). Addition versus deletion as a signal. Animal Learning \& Behavior, 17, 120-133.

Jenkins, H. M., \& Sainsbury, R. S. (1969). The development of stimulus control through differential reinforcement. In N. J. Mackintosh \& W. K. Honig (Eds.), Fundamentalissues in associative learning (pp. 123161). Halifax, NS: Dalhousie University Press.

KlaYman, J., \& HA, Y. (1987). Confirmation, disconfirmation, and information in hypothesis testing. Psychological Review, 94, 211-228.

Lebowitz, B. K., \& Brown, M. F. (1999). Sex differences in spatial search and pattern learning in the rat. Psychobiology, 27, 364-371.

Miller, R. R., \& SPEAR, N. E. (Eds.) (1985). Information processing in animals: Conditioned inhibition. Hillsdale, NJ: Erlbaum.

Nallan, G. B., Bentley, D. M., Carr, J. F., Lyons, K., Moore, D. S., \& UNDERHILL, T. (1994). Adult humans perform better on addition than deletion problems. Psychological Record, 44, 489-499.

Nisbett, R. W., \& Ross, L. (1980). Human inference: Strategies and shortcomings of social judgement. Englewood Cliffs, NJ: Prentice-Hall.

Olton, D. S., \& Samuelson, R. J. (1976). Remembrance of places passed: Spatial memory in rats. Journal of Experimental Psychology: Animal Behavior Processes, 2, 97-115.

(Manuscript received June 1, 2001;

revision accepted for publication December 3, 2001.) 